\section{Beam Tilting Antenna Using Integrated Metamaterial Loading}

Abdolmehdi Dadgarpour, Behnam Zarghooni, Bal S. Virdee, and

Tayeb A. Denidni

\begin{abstract}
This communication presents a technique to re-direct the radiation beam from a planar antenna in a specific direction with the inclusion of metamaterial loading. The beam-tilting approach described here uses the phenomenon based on phase change resulting from an EM wave entering a medium of different refractive index. The metamaterial $\mathrm{H}$-shaped unit-cel structure is configured to provide a high refractive index which was used to implement beam tilting in a bow-tie antenna. The fabricated unit-cell was first characterized by measuring its S-parameters. Hence, a two dimensional array was constructed using the proposed unit-cell to create a region of high refractive index which was implemented in the vicinity bow-tie structure to realize beam-tilting. The simulation and experimental results show that the main beam of the antenna in the $E$-plane is tilted by 17 degrees with respect to the end-fire direction at $7.3,7.5$, and $7.7 \mathrm{GHz}$. Results also show unlike conventional beam-tilting antennas, no gain drop is observed when the beam is tilted; in fact there is a gain enhancement of $2.73 \mathrm{~dB}$ compared to the original bow-tie antenna at $7.5 \mathrm{GHz}$. The reflection-coefficient of the antenna remains $<-10 \mathrm{~dB}$ in the frequency range of operation.
\end{abstract}

Index Terms - Beam tilting, bow-tie antenna, metamaterial.

\section{INTRODUCTION}

Adaptive antennas with reconfigurable pattern have been extensively deployed in the wireless communication systems in order to improve the transmission quality and increase the channel capacity. In fact base stations of wireless communication systems comprise of antenna arrays that are mounted on towers and high buildings which makes it necessary for the antenna to have main beam titling below the horizon. Main beam titling can be accomplished either electronically or mechanically. Electronic beam steering techniques use agile elements such as electronically controlled transmission-lines [1], varactor diodes [2] and RF micro-electromechanical (MEMS) switches [3]. Unfortunately, these techniques suffer from a noticeable gain drop when the beam is tilted. Although the mechanical beam tilting approach [4] shows a better performance at higher tilt angles, however it requires a complicated mechanical installation framework that increases the system size as well as its weight. Another promising technique reported in [5] and [6] achieves beam steering by pivoting an appropriately shaped dielectric substrate in front of a single fixed feed.

In this case a dielectric substrate is shaped in the form of an elliptical lens that is placed over the antenna to tilt the main beam towards a certain direction. The main disadvantage of this technique is that the dielectric lens is large and bulky. Leaky-wave antennas are also employed for beam tilting applications. Recently, a beam scanning leaky-wave slot antenna using a composite right/left-handed (CRLH) technique was reported in [7]. The antenna has a beam scanning angle between $-31^{\circ}$ to $+52^{\circ}$ in the frequency range from 9.5 to $12.4 \mathrm{GHz}$.
However, the tilt angle is a function of the frequency which restricts the application of the antenna. Another drawback of this antenna is its bulky structure resulting from the three-dimensional waveguide framework.

Recently, metamaterials have attracted considerable attention in the light of their unique properties and unprecedented applications in changing the effective properties of materials. Metamaterials have been applied in different antenna applications to realize e.g., bandwidth enhancement, beam focusing, gain enhancement, and polarization efficiency [8], [9].

In this communication, we propose a new solution for beam-tilting applications, in which an integrated metamaterial loading (IML) is implemented directly onto the antenna. This integrated metamaterial loading results in no impact on the antenna's profile, and exhibits necessary refractive index to re-direct its beam.

In the following sections, we first describe the proposed H-shaped metamaterial unit-cell structure, and extract its characterizing parameters. Then a 2D array of the unit-cell is applied on the bow-tie antenna. Finally, the tilting effect of IML on the antenna's main beam is investigated. To validate the results, a prototype of the new IML structure was fabricated, and its S-parameters are measured using a modified version of the free space measurement (FSM) method described in [10]. Then, the characterizing parameters of the media are extracted using a well-known algorithm described in [11]. Finally, two bow-tie antennas with and without the IML structure are fabricated and their return-loss and radiation patterns measurements are presented. The unit-cell's refractive-index is verified over the antenna's operating frequency range. The measured results show the main beam of the antenna is tilted by 17 degrees in the E-plane, i.e., towards the direction of the metamaterial inclusion. The results also show unlike conventional tilted-beam antennas, there is no gain drop when the beam is tilted. In fact, there is a $2.73 \mathrm{~dB}$ increase in the gain of the antenna at $7.5 \mathrm{GHz}$.

\section{BEAM TILTING MEChANiSM}

The mechanism of beam-tilting involving two layered media that are deployed in the antenna is explained in this section. This approach was used to develop a low-profile antenna with a tilted main beam. In Fig. 1 an electromagnetic (EM) point source is placed in the vicinity of a two-layered medium with two different refractive indexes. The EM wave that exits in each layer undergoes a different phase shift. The scenario depicted in Fig. 1 is analogous to a two-element antenna array with a phase shift of $\beta$ between the elements, which is given by:

$$
\beta=k_{o}\left(n_{2}-n_{1}\right) d
$$

The relation between $\beta$ and the radiation angle $\varphi$, which is derived from the antenna theory [12], is given by:

$$
\beta=k_{o} l \sin \varphi
$$

Therefore, from (1) and (2) the tilt angle is given by:

$$
\varphi=\sin ^{-1}\left[\frac{d\left(n_{2}-n_{1}\right)}{l}\right]
$$

According to (3) the tilt angle of the beam is determined by the difference in the refractive index of the two media and its dimensions. As we intend to use this technique for an end-fire planar bow-tie antenna, the above two layered media needs to be located on the substrate of the 


$$
\beta_{1}=\mathrm{k}_{0} \mathrm{n}_{1} \mathrm{~d} \quad \beta_{2}=\mathrm{k}_{0} \mathrm{n}_{2} \mathrm{~d}
$$

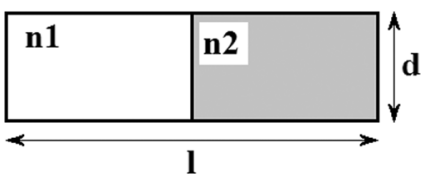

Fig. 1. Mechanism of beam tilting using two layered dielectric media.

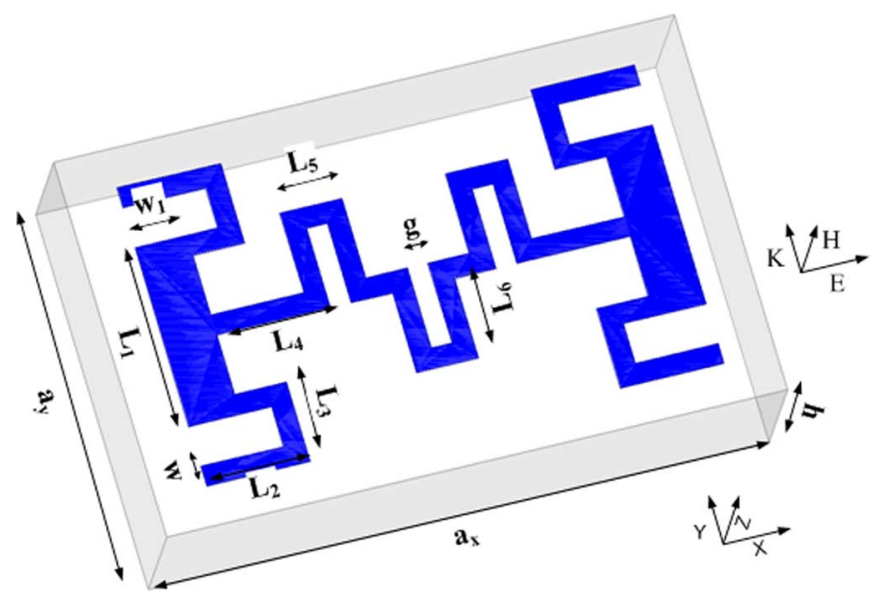

Fig. 2. Geometry of the proposed unit-cell implemented on a dielectric substrate.

antenna. The best solution for changing the refractive index of a limited region of the antenna's substrate was to load the antenna with a suitably designed metamaterial unit-cell. The unit-cell had to (1) provide a high refractive index compared to the substrate, and (2) could be directly integrated onto the dielectric substrate without affecting the antenna's profile. The next section describes the unit-cell structure and its characterizing parameters, which were validated with measured results.

\section{Metamaterial Unit-Cell}

The proposed metamaterial unit-cell structure, shown in Fig. 2, is in fact a modified version of the $\mathrm{H}$-shaped resonator reported in [13]. The difference between the $\mathrm{H}$-shaped resonator and the unit-cell presented here is that the structure includes meandered lines and whose thickness has been modified to realize a higher refractive index. This structure was chosen because it can be easily integrated within the planar bow-tie antenna. The geometry of the antenna determines the polarization of the EM wave which is along the $\mathrm{x}$-direction; it was therefore necessary to design an electric resonator so as to take advantage of the unit-cells' resonance.

The H-shaped meandered line structure was fabricated on a Rogers RT5880 substrate with the thickness of $h=1.575 \mathrm{~mm}$, permittivity of 2.2 , and loss-tangent of 0.0009 . The dimensions of the unit-cell structure are: $\mathrm{a}_{\mathrm{x}}=6 \mathrm{~mm}, \mathrm{a}_{\mathrm{y}}=3.77 \mathrm{~mm}, \mathrm{w}=0.2 \mathrm{~mm}, \mathrm{w}_{1}=0.5 \mathrm{~mm}$, $\mathrm{L}_{1}=1.8 \mathrm{~mm}, \mathrm{~L}_{2}=1 \mathrm{~mm}, \mathrm{~L}_{3}=0.8 \mathrm{~mm}, \mathrm{~L}_{4}=1.1 \mathrm{~mm}, \mathrm{~L}_{5}=0.6$ $\mathrm{mm}, \mathrm{L}_{6}=0.8 \mathrm{~mm}, \mathrm{~g}=0.2 \mathrm{~mm}$.

The unit-cell was simulated using HFSS with PEC and PMC boundary conditions applied to yz and xy-planes, and the two ports were located in Y-direction. S-parameters $\mathrm{S}_{11}$ and $\mathrm{S}_{12}$ of the unit-cell structure were simulated, and its characterizing parameters were extracted using the algorithm described in [11]. To validate the simulated
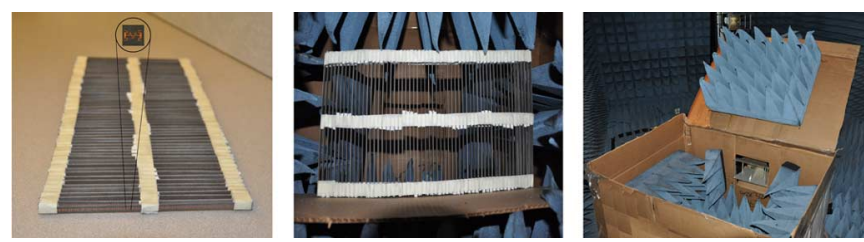

Fig. 3. Measurement setup for acquiring the S-parameters of the unit-cell array.

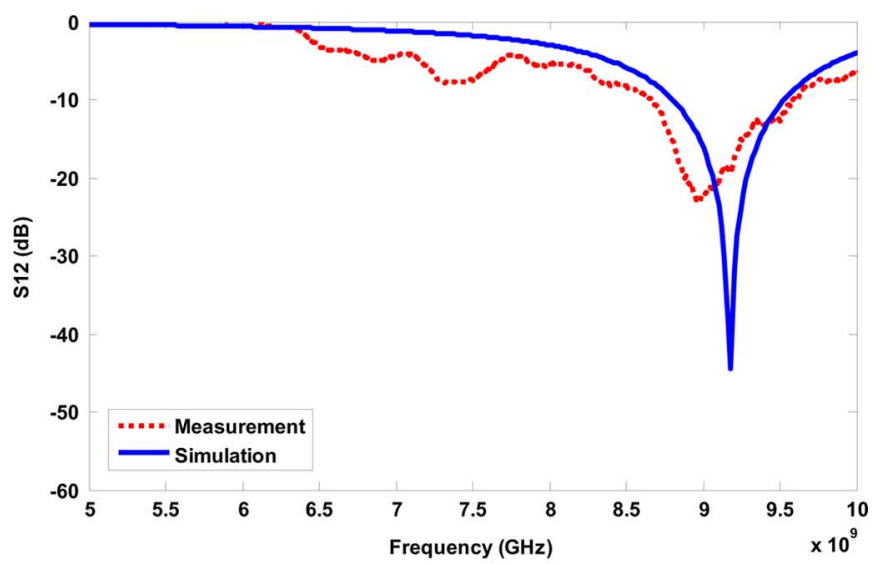

(a)

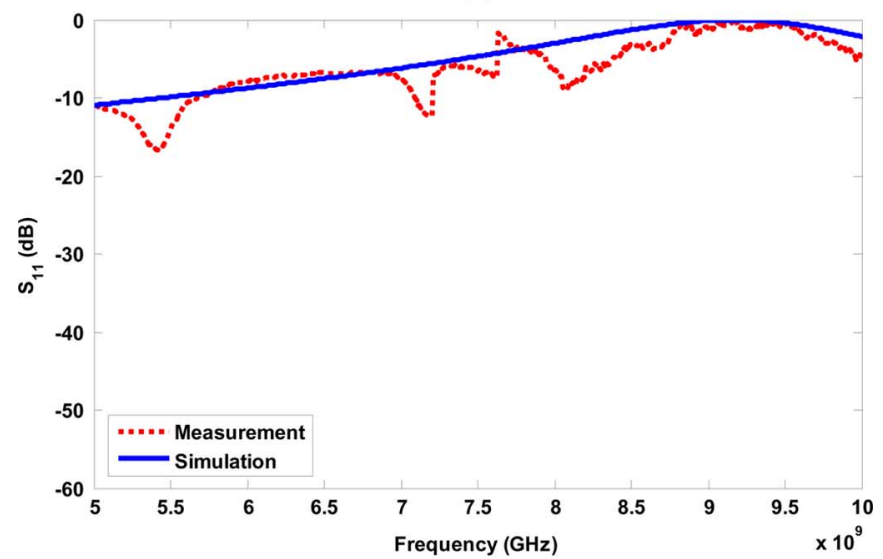

(b)

Fig. 4. The S-parameters of proposed unit-cell structure.

S-parameters, the measurement setup in Fig. 3 was used. In this setup, which is a modified version of the free space measurement (FSM) method in [10], a box was designed to contain a $30 \times 35$ array of the unit-cell, as well as two standard horns acting as transmitter and receiver antennas. Fig. 3 shows a photo of the fabricated array of the proposed unit-cell.

The simulated and measured S-parameters of the unit-cell are shown in Fig. 4(a) and (b). These results were used to extract the array's effective relative permittivity, permeability and refractive-index.

Fig. 5 shows the extracted aforementioned parameters from the measurement results. The results show the unit-cell has a high permittivity (epsilon) and low permeability (mu) behavior across the frequency range $7-8 \mathrm{GHz}$, while the magnitude of the refractive-index varies between 2.1 and 2.4. The actual substrate's refractive index $n_{\text {sub }}$ is 1.6. By applying the metamaterial unit-cell we have effectively increased the refractive index for a portion of the substrate. The proposed meandered line H-shaped unit-cell is compact in size as well as exhibits a relatively high refractive index compared to the substrate over a wideband operating range compared to its $\mathrm{H}$-shaped counterpart in [13]. 


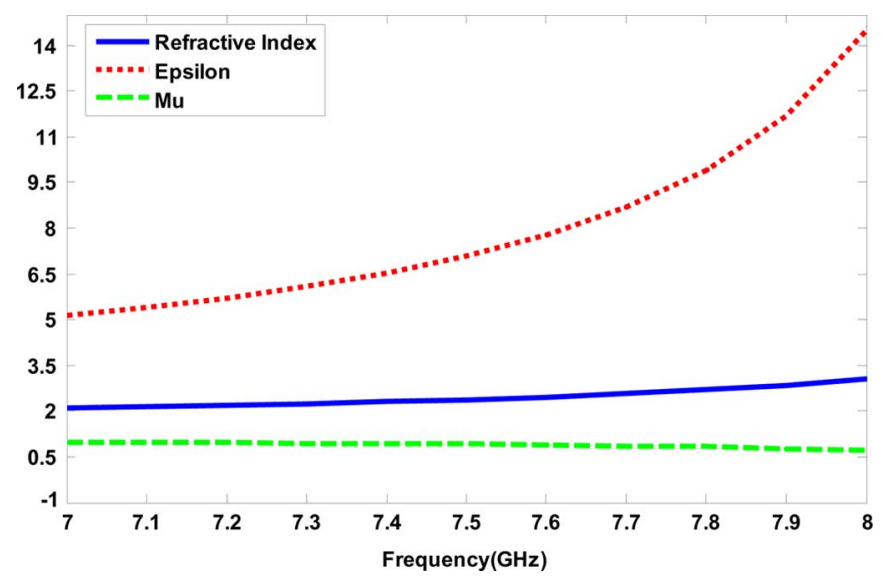

Fig. 5. Retrieved parameters (n, $\varepsilon$, and $\mu$ ) of meandered-line H-shape unit-cell.

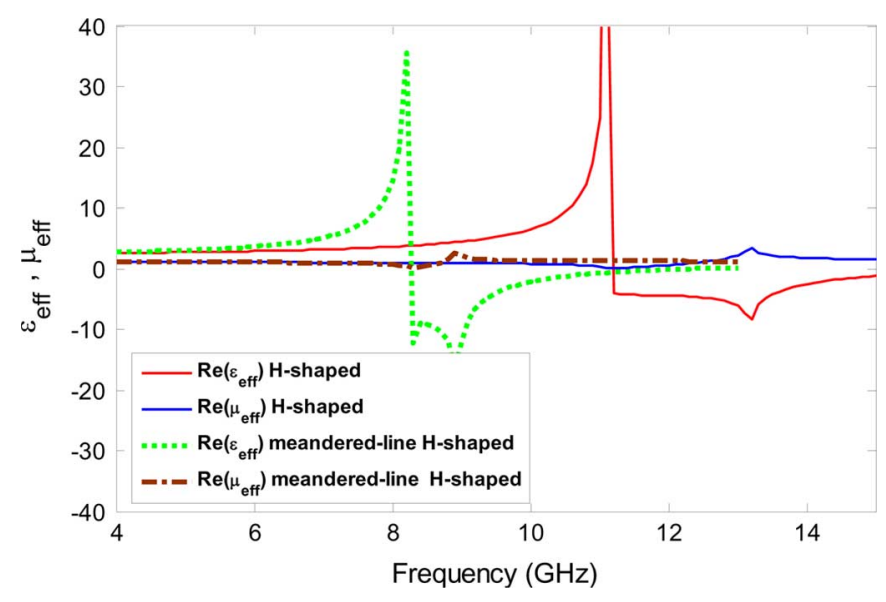

Fig. 6. The extracted permittivity and permeability of the H-shaped and proposed meandered-line unit-cell.

Fig. 6 compares the meandered line unit-cell to its equivalent $\mathrm{H}$-shaped resonator.

Fig. 6 shows the frequency of the permittivity spike shifts from 11.2 $\mathrm{GHz}$ to $8 \mathrm{GHz}$, which corresponds to a miniaturization factor of 0.7 for the proposed unit-cell structure compared to the conventional $\mathrm{H}$-shaped resonator in [13]. Also across the frequency range of 7-8 GHz, which is the operating range of the antenna, we can see that the effective permittivity of the structure is about 7.5, which is nearly twice as higher than the effective permittivity of the conventional $\mathrm{H}$-shaped structure.

\section{BOW-TIE ANTENNA With IML}

Fig. 7 depicts the layout of the proposed antenna with metamaterial unit-cells integrated on the same substrate.

In this design, the reference antenna was a three-element array of bow-tie radiators connected to a microstrip feed-line [14]. A $3 \times 4$ array of the proposed metamaterial unit-cells were printed on the antenna's substrate and located in front of the antenna. The integrated metamaterial loading (IML) on the antenna was printed on a Rogers RT5880 substrate with thickness of $1.575 \mathrm{~mm}$. The dimensions of the proposed antenna are: $\mathrm{a}=9.2 \mathrm{~mm}, \mathrm{~b}=11 \mathrm{~mm}, \mathrm{c}=8.4 \mathrm{~mm}$, $\mathrm{k}=10.8 \mathrm{~mm}, \mathrm{e}=28 \mathrm{~mm}, \mathrm{f}=4.4 \mathrm{~mm}, \mathrm{R}_{1}=7.9 \mathrm{~mm}, \mathrm{R}_{2}=5.4$ $\mathrm{mm}, \mathrm{R}_{3}=3.6 \mathrm{~mm}$. Coupling between the IML and the antenna is through electromagnetic interaction. Fig. 8 shows the radiation pattern of the bow-tie antenna with and without IML structure in the E-plane of the antenna.

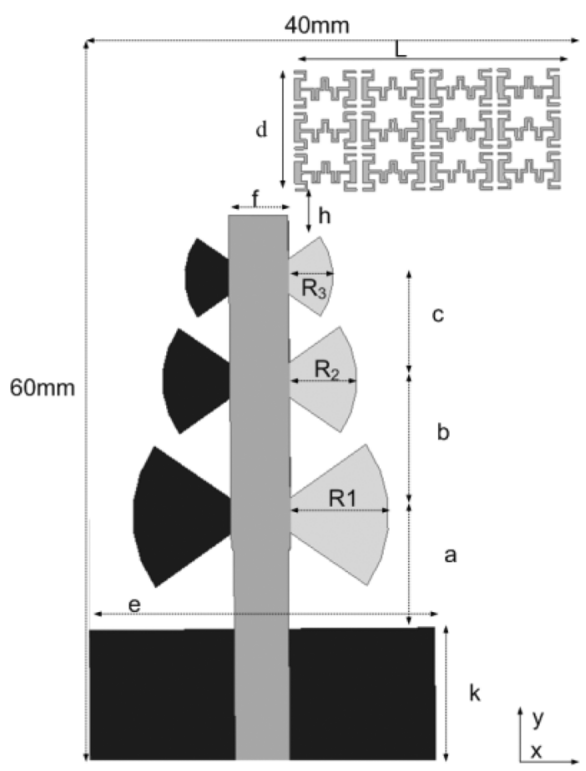

Fig. 7. Configuration of proposed antenna embedded with IML unit-cells on the top surface of substrate.

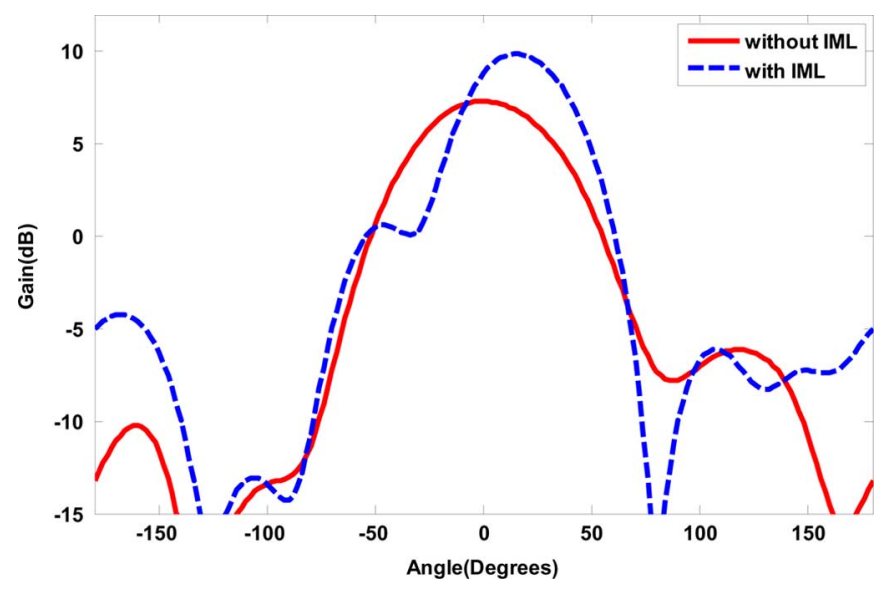

Fig. 8. Radiation patterns of proposed antenna in the E-plane (xy) with IML unit-cells and a conventional bow-tie antenna at $7.5 \mathrm{GHz}$.

Fig. 8 shows that when the IML is included in the antenna substrate the main beam of the antenna is tilted by an angle of 17 degrees. The tilt angle can be calculated using (3), where $\mathrm{n}_{2}=\mathrm{n}_{\mathrm{IML}}=2.2$, $\mathrm{n}_{1}=\mathrm{n}_{\text {sub }}=1.6, \mathrm{~d}=9.7 \mathrm{~mm}$ and $l=21.5 \mathrm{~mm}$ which gives us $\varphi=17$. Fig. 9(a) and (b) shows the Poynting vector and electrical-field distribution over the antenna structure with and without IML inclusion, respectively, at $7.5 \mathrm{GHz}$. These figures show that when there is no IML the antenna's energy is radiated predominately in a vertical direction, but when the IML inclusion is added to the structure the radiation is tilted towards the location of the IML.

According to Fig. 9(c) and (d), the distribution of the electrical-field is completely modified in presence of the IML structure. The modified E-field induces current in the unit-cells that makes it behave like a parasitic element to re-direct the antenna's main beam as well as enhancing its gain [12].

\section{PARAMETRIC STUDY}

This section describes the results of a study to determine how different numbers of unit-cells affect the tilt angle performance in the 


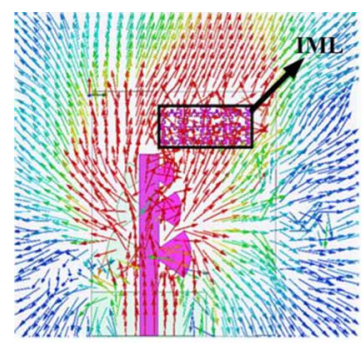

(a)

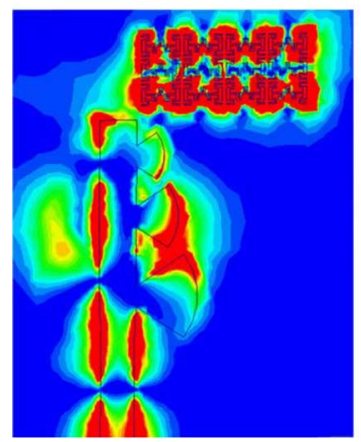

(c)

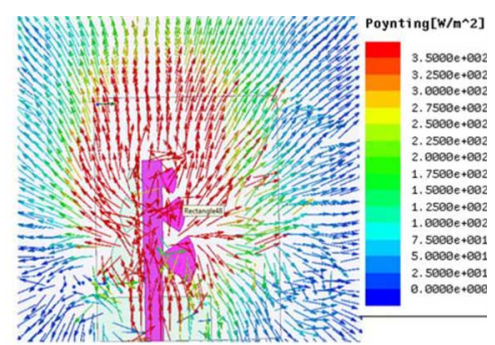

(b)

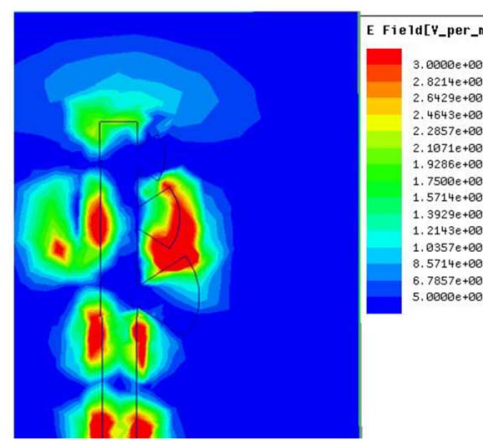

(d)
Fig. 9. Poynting vector and E-field distribution over the antenna at $7.5 \mathrm{GHz}$ : (a) \& (c) with IML, and (b) \& (d) without IML.

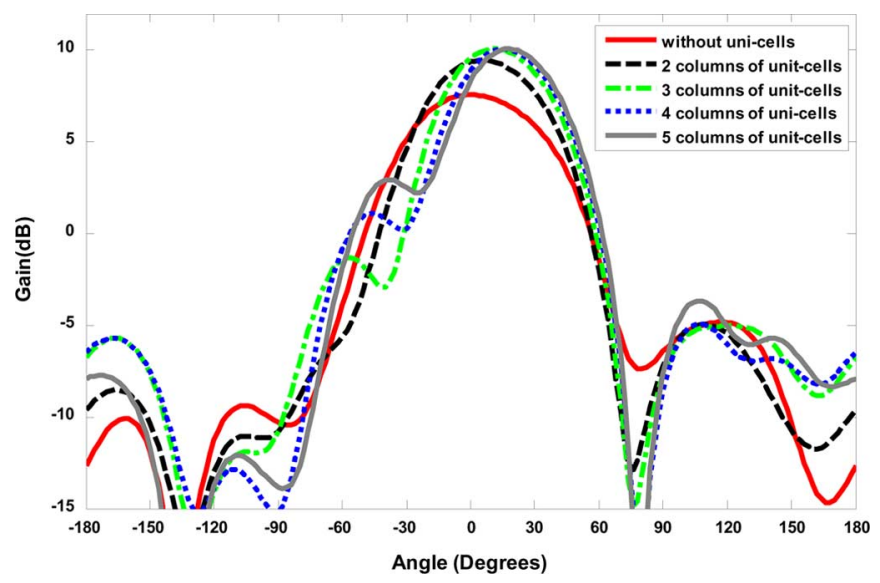

Fig. 10. Radiation pattern of proposed antenna with different columns of unitcell loading in the y-direction.

E-plane. In this study, the antenna was simulated with an IML inclusion having 2, 3, 4 and 5 columns, and in each case the effect of the IML on gain, main beam directivity, and side-lobe level (SLL) of the antenna was investigated.

Fig. 10 shows the radiation pattern of the antenna in the E-plane (xy) at $7.7 \mathrm{GHz}$ for various levels of unit-cell loading. The results clearly show the incorporation of the IML structure increases the gain of the antenna. Gain enhancement is due to the parasitic effect of unit-cells printed on the substrate which act as parasitic directors. These results are summarized in Table I.

It is clear from Table I that when the number of the unit-cell columns is increased a higher tilt angle is observed. However, there is a trade-off between the tilt angle and the SLL, and as we can see from Table I that with 5 columns the SLL is $-7 \mathrm{~dB}$, which is unacceptable. The other effect of the IML is gain enhancement, which increases with increase in the number of the columns. The parametric study in this case shows the best performance of the antenna can be achieved when four columns are used in the structure of IML. Another parameter that was investigated
TABLE I

THE EFFECT OF IML ON ANTENNA PERFORMANCE

\begin{tabular}{|c|c|c|c|c|}
\hline $\begin{array}{c}\text { No. of unit-cell columns } \\
\text { in IML structure }\end{array}$ & 2 & 3 & 4 & 5 \\
\hline Tilt angle (degree) & 6 & 12 & 17 & 19 \\
\hline Gain (dB) & 9.45 & 10.07 & 10.05 & 10 \\
\hline Side-lobe level (dB) & -14.5 & -11.38 & -9 & -7 \\
\hline
\end{tabular}

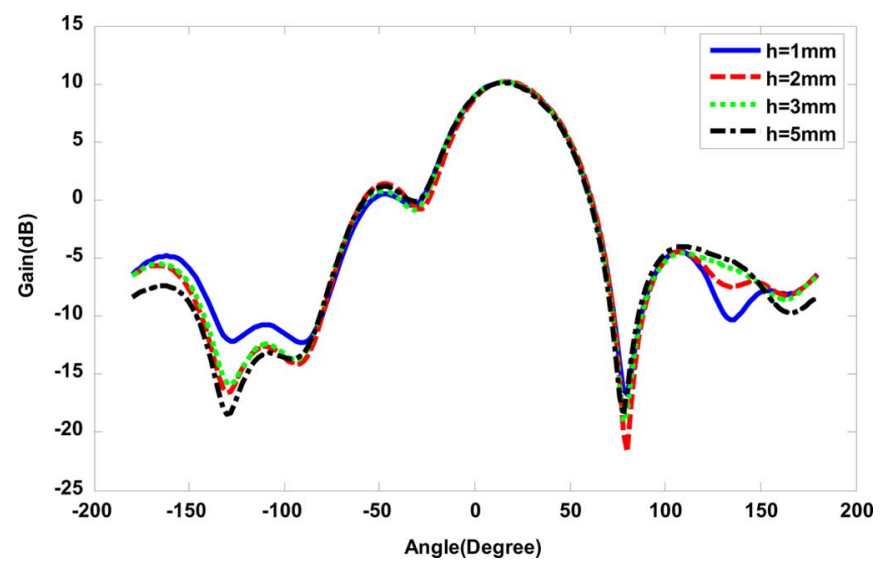

Fig. 11. The radiation pattern of antenna in E-plane as a function of parameter h.

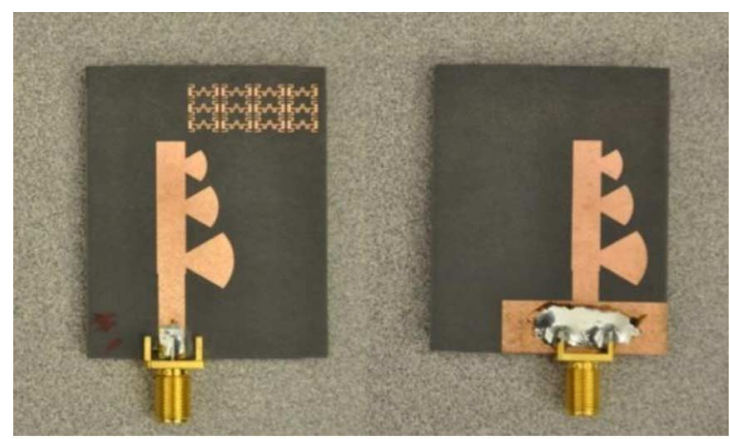

(a)

(b)

Fig. 12. Photograph IML antenna, (a) top view, (b) bottom view.

was the distance $(h)$ between IML and the end bow-tie radiator, as shown in Fig. 7. In this study, $h$ was varied from $1 \mathrm{~mm}$ to $5 \mathrm{~mm}$, and the resulting radiation patterns are shown in Fig. 11. These results indicate that the distance $h$ does not have any significant effect on the beam tilting angle.

The parametric study shows that the main beam angle can be tilted from 6 to 19 degrees when the IML columns are increased from 2 to 5. Therefore, if a reconfigurable IML is used in the antenna's structure the main beam can be steered from 6 to 19 degrees. A reconfigurable structure can be realized using active elements such as PIN diodes, and investigation of this is under progress.

\section{EXPERIMENTAL RESULTS}

The proposed antenna design with the embedded IML structure was validated practically. The bow-tie antenna with a $3 \times 4$ array of meander-line metamaterial unit-cells were fabricated and measured. A photograph of the fabricated prototype is shown in Fig. 12.

The measured and simulated reflection-coefficient of the antenna with and without IML inclusion is shown in Fig. 13. The reflection-coefficient is altered in presence of the IML, which is due to the mutual 


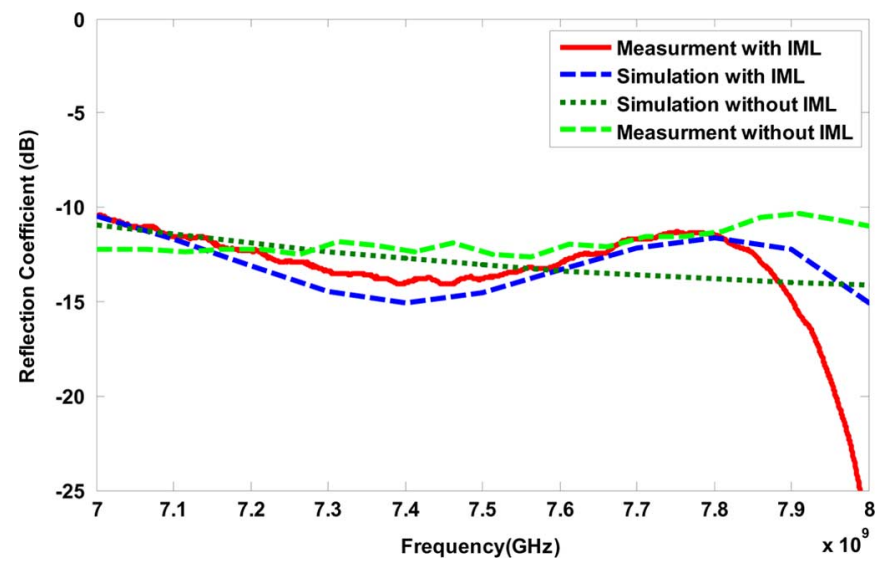

Fig. 13. Reflection-coefficient of bow-tie antenna loaded with IML.

TABLE II

Measured Peak Gain of Antenna at Different Scan Angles

\begin{tabular}{|c|c|c|c|c|}
\hline $\begin{array}{c}\text { Freq. } \\
(\mathrm{GHz})\end{array}$ & $\begin{array}{c}\text { Simulated } \\
\text { peak gain } \\
\text { without IML }\end{array}$ & $\begin{array}{c}\text { Measured } \\
\text { peak gain } \\
\text { without IML }\end{array}$ & $\begin{array}{c}\text { Simulated } \\
\text { peak gain } \\
\text { with IML at } \\
17^{\circ}\end{array}$ & $\begin{array}{c}\text { Measured } \\
\text { peak gain } \\
\text { with IML at } \\
17^{\circ}\end{array}$ \\
\hline 7.3 & 6.44 & 5.71 & 8.9 & 8.7 \\
\hline 7.5 & 7 & 6.5 & 9.5 & 9.23 \\
\hline 7.7 & 7.40 & 7.84 & 10 & 9.25 \\
\hline
\end{tabular}

coupling effect between the bow-tie radiators and the unit-cells. However, the magnitude of $\mathrm{S}_{11}$ remains lower than $-10 \mathrm{~dB}$ in the frequency range of 7-8 GHz.

The simulated and measured normalized radiation patterns of the proposed antenna with IML structure in the E-plane are plotted in Fig. 14(a)-(c) at 7.3, 7.5, and 7.7 GHz. Fig. 14(d) shows the simulated and measured radiation pattern of the antenna in the H-plane at 7.5 $\mathrm{GHz}$, where the main beam is oriented in the end-fire direction without tilting.

The peak gain measurement at different frequencies was carried out using the gain comparison method with a known standard horn antenna, which can be calculated using the following expression:

$$
G_{\mathrm{AUT}}=G_{\mathrm{GS}}+10 \log \left(\frac{P_{\mathrm{AUT}}}{P_{\mathrm{GS}}}\right)
$$

where $G_{\mathrm{AUT} \mathrm{dB}}$ is the gain of IML bow-tie antenna, and $G_{\mathrm{GS} \mathrm{dB}}$ is the gain of standard horn antenna with specification of EMCO model operating in the frequency range of $5.85-8.2 \mathrm{GHz}$. The measured peak gain can be obtained by calculating the receiving power of the antenna under test $\left(P_{\mathrm{AUT}}\right)$ and the standard horn's power in receiving mode $\left(P_{\mathrm{GS}}\right)$. From the results given in Table II the measured gain of the proposed antenna with IML at $7.5 \mathrm{GHz}$ is $9.23 \mathrm{~dB}$, which is $2.73 \mathrm{~dB}$ more than the conventional bow-tie antenna.

\section{CONCLUSION}

A novel technique is presented that uses metamaterial unit-cell loading, which is implemented onto the planar antenna, in the vicinity of radiating element to re-direct its main beam in a specific direction. The antenna employed comprises of a three-element array of bow-tie radiators, and the beam tilting method employed is based on the application of metamaterial inclusions to realize a region of high refractive index. The metamaterial unit-cell structure was characterized and its behavior and performance including its high refractive-index

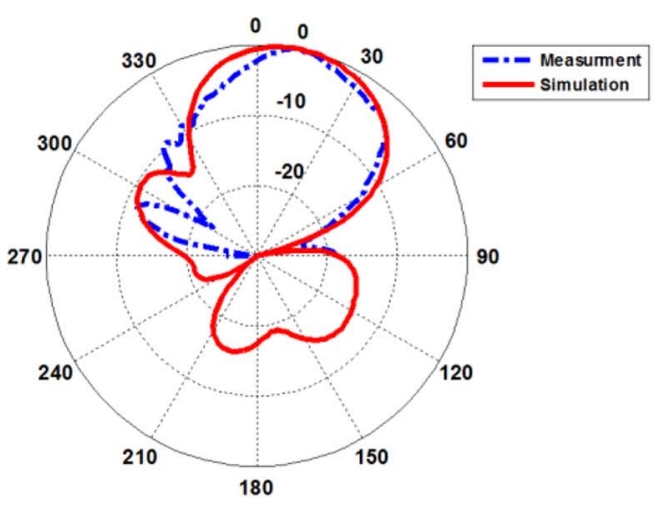

(a)

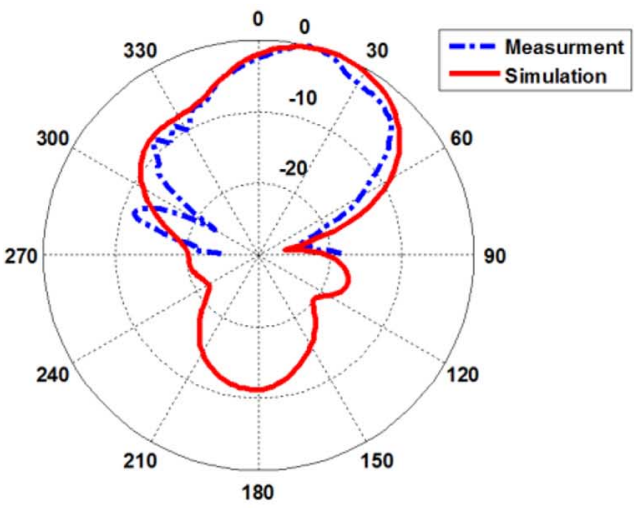

(b)

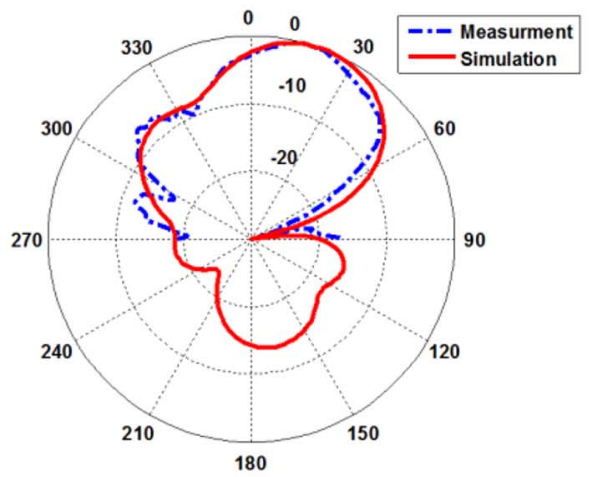

(c)

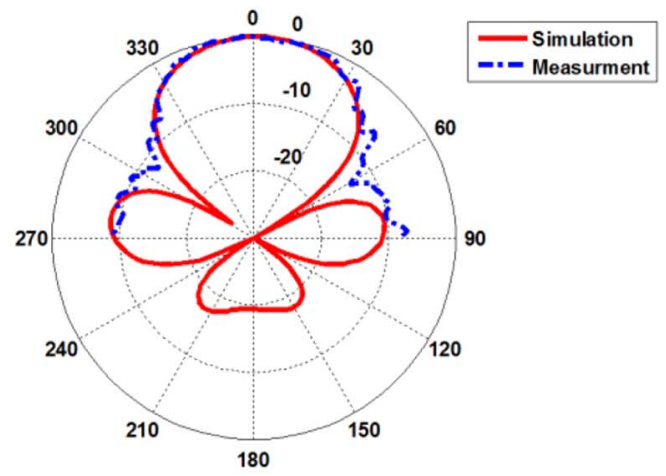

(d)

Fig. 14. Radiation pattern of proposed antenna with IML at: (a) $7.3 \mathrm{GHz}$, (b) $7.5 \mathrm{GHz}$, (c) $7.7 \mathrm{GHz}$, and (d) with IML in the H-plane at $7.5 \mathrm{GHz}$.

was verified through fabrication and measurement. Compared to a conventional $\mathrm{H}$-shaped resonator the proposed unit-cell provides a miniaturization factor of 0.7 as well as a higher refractive-index. A 
$3 \times 4$ array of metamaterial unit-cell loading was integrated within a bow-tie antenna. The simulation and experimental results show that the antenna's main beam tilted by 17 degrees in a direction towards the location of the metamaterial loading in the E-plane, while the H-plane radiation pattern remains unchanged. The tilt angle observed correlates with the predicted value. Unlike other conventional methods of tilting, the method described here results in no degradation in gain, in fact a gain enhancement of $2.73 \mathrm{~dB}$ is observed, which is due to parasitic behavior of the metamaterial unit-cell inclusion.

\section{REFERENCES}

[1] S. Lim, C. Caloz, and T. Itoh, "Metamaterial-based electronically-controlled transmission line structure as a novel leaky-wave antenna with tunable radiation angle and beamwidth," IEEE Trans. Microw. Theory Tech., vol. 53, no. 1, pp. 161-173, Jan. 2005.

[2] J. J. Luther, S. Ebadi, and X. Gong, "A microstrip patch electronically steerable parasitic array radiator (ESPAR) antenna with reactance-tuned coupling and maintained resonance," IEEE Trans. Antennas Propag., vol. 60, no. 4, pp. 1803-1813, Sep. 2012.

[3] E. R. Brown, "RF-MEMS switches for reconfigurable integrated circuits," IEEE Trans. Microw. Theory Tech., vol. 46, no. 11, pp. 1868-1880, Nov. 1998.

[4] J. Huang and A. C. Densmore, "Microstrip Yagi antenna for mobile satellite vehicle application," IEEE Trans. Antennas Propag., vol. 39, no. 7, pp. 1024-1030, Jul. 1991.
[5] D. F. Filipovic, G. P. Gauthier, S. Raman, and G. M. Rebeiz, "Off-axis properties of silicon and quartz dielectric lens antennas," IEEE Trans. Antennas Propag., vol. 45, no. 5, pp. 760-766, May 1997.

[6] J. R. Costa, E. B. Lima, and C. A. Fernandes, "Compact beam-steerable lens antenna for $60-\mathrm{GHz}$ wireless communications," IEEE Trans. Antennas Propag., vol. 57, no. 10, pp. 2926-2933, Oct. 2009.

[7] D.-J. Kim and J.-H. Lee, "Beam scanning leaky-wave slot antenna using balanced CRLH waveguide operating above the cutoff frequency," IEEE Trans. Antennas Propag., vol. 61, no. 5, pp. 2432-2440, May 2013.

[8] M. A. Antoniades and G. V. Eleftheriades, "Multiband compact printed dipole antennas using NRI-TL metamaterial loading," IEEE Trans. Antennas Propag., vol. 60, no. 12, pp. 5613-5626, Dec. 2012.

[9] D. Li, Z. Szabó, X. Qing, E.-P. Li, and Z. Chen, "A high gain antenna with an optimized metamaterial inspired superstrate," IEEE Trans. Antennas Propag., vol. 60, no. 12, pp. 6018-6023, Dec. 2012.

[10] U. C. Hasar and J. J. Barroso, "Permeability measurement of metamaterials with split-ring-resonators using free-space calibration-independent methods," J. Infrared, Milli., Terahertz Waves, vol. 33, no. 2, pp. 218-227, Feb. 2012.

[11] X. Chen, T. M. Grzegorczyk, B. I. Wu, J. Pacheco, Jr., and J. A. Kong, "Robust method to retrieve the constitutive effective parameters of metamaterials," Phys. Rev. Lett., vol. E 70, p. 016608, 2004.

[12] C. A. Balanis, Antenna Theory: Analysis and Design, 3rd ed. New York, NY, USA: Wiley, 2005.

[13] R. Liu, C. Ji, J. J. Mock, J. Y. Chin, T. J. Cui, and D. R. Smith, "Broadband ground-plane cloak," Science, vol. 323, no. 5912, pp. 366-369, Jan. 2009.

[14] S.-W. Qu, J.-L. Li, Q. Xue, and C.-H. Chan, "Wideband periodic endfire antenna with bowtie dipoles," IEEE Antennas Wireless Propag. Lett., vol. 7, pp. 314-317, 2008. 